\title{
HISTORIA
}

\section{LA ENFERMERÍA ANTROPOLÓGICA Y \\ TRANSCULTURAL EN EL MARCO DE LA \\ EDUCACIÓN PARA EL DESARROLLO. UNA PERSPECTIVA HISTÓRICA Y EPISTEMOLÓGICA EN EL UMBRAL DEL SIGLO XXI}

\author{
José Siles González*, Encarnación García Hernández**, Luis Cibanal Juan*, \\ Roberto Galao Malo***
}

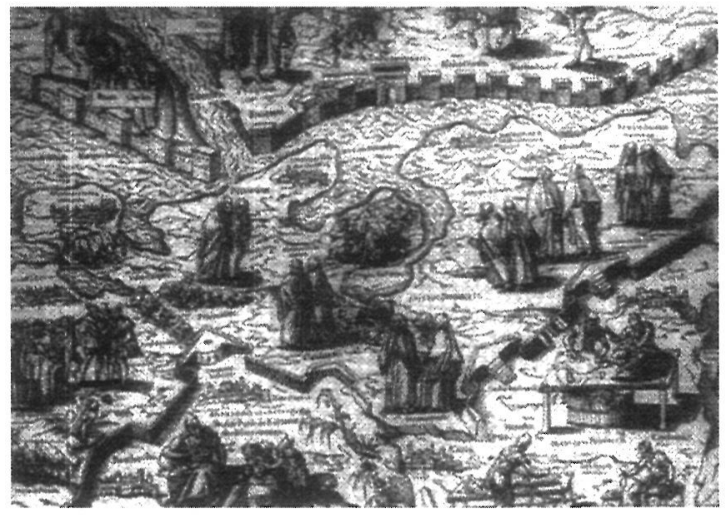

\section{RESUMEN}

El objetivo de este trabajo consiste en clarificar históricamente el concepto de "desarrollo" y vincularlo a otro concepto, el de "educación"con la finalidad de aportar un marco idóneo para la acción educativa en enfermería, especialmente en enfermería antropológica y transcultural. Para ello se han identificado los significados de los conceptos implicados separada y conjuntamente procurando interrelacionar los factores sociales, ideológicos, económicos, políticos, científicos y metodológicos. Asimismo, una vez analizados y contextualizados los diferentes conceptos, atendiendo a su naturaleza, se ha procedido a seleccionar las técnicas y métodos idóneos para el trabajo en educación para el desarrollo y enfermería transcultural. Entre las conclusiones cabe destacar la importancia de la contextualización de todas las actividades de educación para el desarrollo, la necesidad de adoptar métodos y técnicas adecuados al con- texto de la acción educativa en enfermería. Por otro lado se ha constatado que tanto la enfermería como la educación para el desarrollo comparten el carácter holístico con el que abordan su objetosujeto: el hombre en el proceso de satisfacción de necesidades. Los métodos y técnicas de investigación acción resultan ser los más adecuados para este tipo de trabajos debido, sobre todo a la repercusión de la actividad investigadora en la realidad investigada (no se agota el proceso científico en la producción de conocimientos). Tanto la enfermería para el desarrollo, como la enfermería comunitaria y la enfermería transcultural comparte el principio esencial y característico de tales disciplinas: el fomento de la participación en un clima de tolerancia y solidaridad. No es posible desarrollar ninguna actividad de educación para el desarrollo, enfermería comunitaria o enfermería transcultural sin la implicación previa de los individuos, las familias o las comunidades a las que vayan dirigidas.

Palabras clave: Educación para el desarrollo, pedagogía activa, investigación acción, educación para la salud, pedagogía de los cuidados.

ANTHROPOLOGICAL AND TRANSCULTURAL NURSING IN THE CONTEXT OF EDUCATION FOR DEVELOPMENT: A HISTORICAL AND EPISTEMOLOGIC PERSPECTIVE IN THE PRINCIPLE OF THE XXI CENTURY 


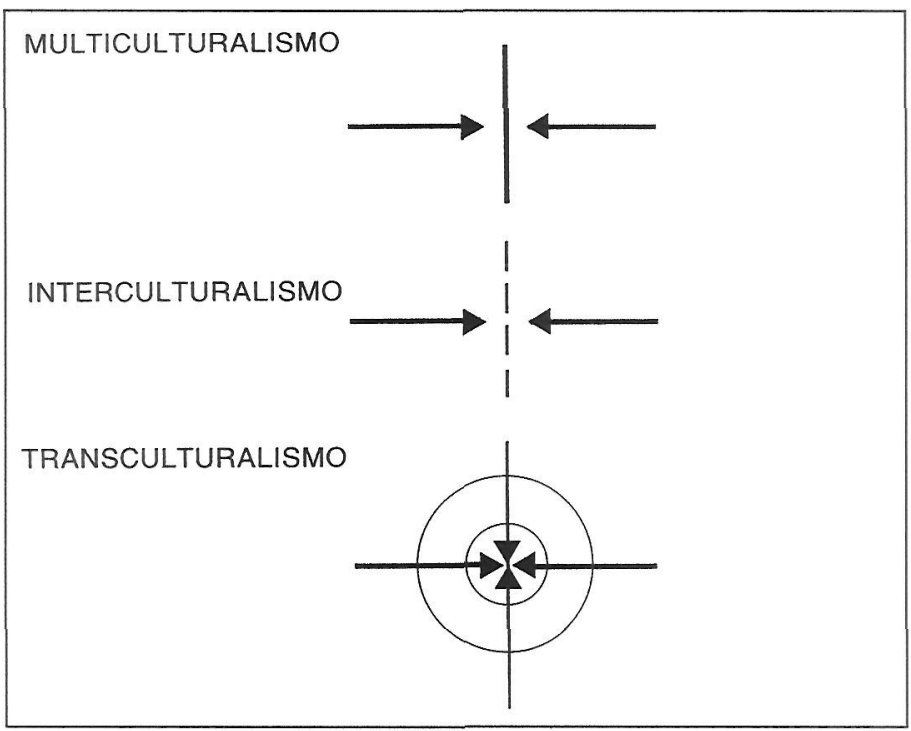

\section{ABSTRACT}

This work has the objective of clarify the development concept historically and relative it with another concept: education; and everything this process with the purpose of contributing to selection of a suitable context for educational nursing acction, specially in anthropological and transcultural nursing.

Key words: Transcultural nursing, development, education.

\section{INTRODUCCIÓN}

En este trabajo se aborda el complejo tema del desarrollo y su relación con la educación y todo ello con la enfermería comunitaria, la antropología de los cuidados y la enfermería transcultural. Para todo ello, en primer lugar, se ha realizado un análisis histórico de la evolución de los conceptos de desarrollo y educación, tarea del todo imposible sin considerar la influencia de otros factores: la política y la ideología científica. La finalidad de este trabajo de clarificación histórica, conceptual y hermenéutica ha consistido en alcanzar niveles aceptables de contextualización histórica de los conceptos mencionados y la relación de sus diferentes interpretaciones con el papel desempeñado por la enfermería comunitaria y transcultural. La reflexión sobre las líneas maestras que han vinculado tanto los conceptos de "desarrollo" y "educación" como sus correspondientes mutaciones, ha ocupado una de las partes más destacadas de este proceso. Metodológicamente, pues, se parte de criterios derivados de la investigación cualitativa en la que se han procedido a la elaboración de una serie de cuestiones previas que han orientado el proceso desde su principio hasta las conclusiones favoreciendo un clima de reflexión crítica del grupo de expertos. En primer lugar se han planteado cuestiones centrales tales como:

- ¿Cuándo y porqué surge el concepto de educación para el desarrollo?

- ¿Qué cariz ha tomado en cada momento histórico la educación como instrumento fundamental de la filosofía del desarrollismo?

- ¿Ha influido la política bipolar de bloques en una diferenciación teórico-práxica del desarrollo?

- ¿Qué papel puede desempeñar la enfermería en el marco de la educación para el desarrollo?

- ¿Qué clase de métodos y técnicas de enfermería resultan más apropiados en educación para el desarrollo?

- ¿Los usos y procedimientos científicos están influidos por las ideologías?

- Qué relación existe entre el sistema político de un país y la posibilidad de desarrollar la enfermería comunitaria y transcultural.

- ¿Qué relación existe entre los paradigmas científicos, la educación para el desarrollo y la enfermería transcultural? 
- ¿Qué analogías y diferencias existen entre enfermería antropológica y enfermería transcultural?

- ¿Qué relaciones existen entre los principios, métodos y prácticas de la educación para el desarrollo, la enfermería comunitaria y la enfermería transcultural?

\section{DESARROLLO DEL TEMA}

\section{EL CONCEPTO DE DESARROLLO}

Para que la existencia del término "desarro1lo" fuera posible, previamente tuvieron que darse algunas condiciones. Entre estos prolegómenos del concepto de desarrollo hay que destacar la concienciación del déficit que existía en algunos países (o dentro de un mismo país) respecto del nivel de vida deseable. Tomando siempre como referencia las metrópolis y cierta nostalgia de sus antiguas colonias, tras la segunda guerra mundial -en cuyo transcurso los bloques contendientes utilizaron los recursos humanos, materiales y espaciales de sus respectivas colonias y "áreas de influencia"- no se puso en cuestión la necesidad de mantener la dependencia de las ex-colonias. En definitiva, tras la paz de Versalles la dominación basada en la ocupación militar parecía caída en la obsolescencia más absoluta y había que inventar otra fórmula para que las políticas y las economías de los países desarrollados siguieran beneficiándose de sus excolonias. Para ello debían sintonizar ciertos elementos fundamentales de las economías -casi inexistentes por exangües- de los esquilmados países subdesarrollados con las nuevas exigencias imperantes en el orden internacional. El hecho de que España perdiera lo que quedaba de sus colonias a finales del siglo XIX, no era un hecho aislado. Las características de Estados Unidos, país que sustituyó a la metrópoli hispana en Cuba, Puerto Rico y Filipinas -sumida en la obsolescencia política, económica y social-, constituyen un paradigma de lo que iba a constituir el nuevo imperialismo (MESA, 1967).

Entre las definiciones volcadas hacia el desarrollo económico destaca la aportada por Organski respecto a la política de desarrollo: "(...) la eficacia creciente del gobierno en utilizar los recursos humanos y materiales de la nación para fines nacionales (...)" (PUELLES, 1987). POSTMAN ofrece una visión histórica del concepto de desarrollo: En la primera fase, tras la II Guerra Mundial, se llegó a la conclusión de que era posible que en toda sociedad subdesarrollada "que contara con la voluntad política necesaria podría adquirir la capacidad de crecer económicamente" (POSTMAN, 1983).En esta primera etapa ya se precisaron algunos de los aspectos que había que cumplir para que se produjera realmente el desarrollo:

- Transferencia del poder social de los grupos dirigentes tradicionales.

- Voluntad de reorganizar la estructura institucional de la sociedad desde la perspectiva de las sociedades desarrolladas.

- Acceso a la ciencia y a la tecnología.

- Adquisición por parte de la población de técnicas y habilidades necesarias para el desarrollo.

Los dos últimos puntos constituyeron el centro de atención para lograr el objetivo deseado. Tanto la transferencia del poder social de los grupos dirigentes como la reorganización de la estructura institucional suponían un escollo de difícil acceso y peor resolución.

En esta primera fase la educación era considerada como un requisito para que se diera el desarrollo (POSTMAN, 1983). Puelles relaciona la estabilidad política con la capacidad para renovar las élites que integran el poder. Asimismo, opina que la mejor garantía para que se pueda producir un relevo continuo de la clase política es el desarrollo del sistema educativo: "(...) el reclutamiento de la élite política no es más que un aspecto más de la formación de las élites (...) el sistema de educación formal permite en las sociedades modernas, en mayor o menor grado, alcanzar el estatus social y, por ende, el estatus político (...)" (PUELLES, 1987).

Las críticas generalizadas en los años sesenta originaron un cambio de tendencia en el tema desarrollista, y dieron lugar a una segunda fase diferenciada. Se desvaneció la creencia de que el crecimiento económico favorecía a todos los grupos sociales por igual. El crecimiento, al contrario de lo esperado, incrementaba el dualismo en la sociedad, por lo que se hacía preciso marcar un nuevo objetivo: la redistribución equitativa del crecimiento económico (POSTMAN, 1983). 
En el terreno educativo esta fase se caracterizó por orientar la educación hacia la totalidad de la población con el objetivo de dotarla de una mínima capacidad organizativa para poder desarrollar sus propios programas productivos. La ausencia de la participación de la población en el proceso de planificación y resolución de sus propios problemas fue, en gran medida, una consecuencia de las circunstancias anteriormente reseñadas. Otra de las críticas correspondientes a esta fase se cifran en la destrucción de la identidad cultural de los pueblos implicados en políticas de desarrollo. Para evitar esta occidentalización a ultranza se planificaron sin alcanzar los resultados esperados- estrategias contextualizadas de desarrollo o desarrollismo contextualizado.

La tercera fase estuvo marcada por la recesión. En la década de los setenta se vivió una crisis que provocó la alerta mundial respecto a las existencias de materias primas y energéticas (especialmente petróleo). En este estado recesivo, los científicos empezaron a centrarse en el nuevo concepto de desarrollo surgido en el ámbito latinoamericano y que sintetiza el punto de vista histórico de dicha cuestión desde el enfoque colonialista o neocolonialista: "(...) el desarrollo es un proceso que tiene lugar a nivel del sistema económico mundial, y no a nivel de las sociedades nacionales individuales, por lo cual queda implícita la necesidad de una transformación de la estructura económica mundial (...)...en otras palabras el desarrollo no puede separarse del subdesarrollo (...)" (POSTMAN, 1983: 1338). Sobre esta visión del subdesarrollo y desarrollo como las dos caras de la misma moneda, Ander Egg señala que los que sostienen estos postulados se basan, fundamentalmente en la historia. La historia de los países desarrollados muestra nítidamente cómo y a costa de qué y de quienes alcanzaron el desarrollo en un contexto económico y político que les favorecía. La historia, asimismo, explica en que medida el desarrollo de los países ricos truncó sus posibilidades de expansión autónoma, explicitando las causas de su actual nivel de dependencia en el orden económico y político (ANDER EGG, 1980).

Finalmente, en una cuarta fase, se llegó a reconocer el estatuto de globalidad del desarrollo. La educación evolucionó en ese mismo sentido ecológico y armonizador: la transnacionalidad y el transculturalismo. El objetivo prioritario de la transnacionalidad consistía en acelerar la formación de las élites y extender progresivamente los distintos escalones del sistema educativo a la totalidad de la población contando con la implicación previa de la misma.

\section{EL PAPEL DE LA CIENCIA EN EL DESARROLLO Y LA EDUCACIÓN DE LA COMUNIDAD}

En este contexto surgió el debate sobre la universalidad de la ciencia, su neutralidad o tendencia ideológica, etc. Bunge en "Ciencia y Desarrollo" aborda diversos temas que afectan a la ciencia y que convergen sobre el plano central de "desarrollo". Tras reafirmar el carácter sistémico de desarrollo científico y sus condiciones generales, se detiene a analizar la relación de la ciencia con los problemas nacionales, se cuestiona si es viable o tan siquiera posible investigar en países subdesarrollados. Finalmente disecciona la anatomía ideológica de la ciencia y su relación con la filosofía como instrumento de búsqueda continua de verdad. Bunge concluye reconociendo el carácter global inherente a todo proceso de desarrollo: "(...) la sociedad humana no es ni un bloque macizo ni un mero conjunto de individuos, sino un sistema concreto analizable en cuatro subsistemas principales: el biológico, económico, cultural y político "(...)" (BUNGE, 1982). Asimismo, Bunge señala que se debe fomentar el desarrollo "in situ" o contextualizado de las ciencias y técnicas, para evitar que los problemas a resolver a estos niveles tengan que ser siempre tratados desde el exterior por investigadores ajenos a la problemática en cuestión (BUNGE, 1982). Sin embargo este autor, después de reconocer que el ritmo de la ciencia es regulado por la política y por la imagen de los científicos, no es muy optimista respecto al futuro de la ciencia ya sea en los países desarrollados o en los subdesarrollados y tras certificar su estado de crisis señala sus causas en ambos contextos internacionales: "(...) la crisis de la ciencia se agudiza en los países desarrollados debido a la crisis energética, a la carrera armamentista y a la ya endémica estanflacción (o inflacción acompañada de estancamiento económico). En los países subdesarrollados sirve de excusa el propio subdesarrollo, 
hay que postergar la ciencia dejándola para cuando seamos prósperos (...) no se puede hacer investigación cuando la mitad de la población no tiene acceso a los servicios más elementales." (BUNGE, 1982: 146-147). Bunge, después de constatar la muerte de la ciencia en dos períodos históricos (la ciencia griega en la época cristiana y la debacle científica bajo la Europa de los dictadores a mediados del siglo XX) vaticina las sorprendentes consecuencias que tendrían para los países desarrollados y subdesarrollados si, finalmente, se dejara de hacer ciencia relegando definitivamente el tema de la investigación: "(...) la muerte de la ciencia sería penosa y acaso fatal para las sociedades de los dos primeros mundos. Pero no afectaría a las tres cuartas partes de la humanidad, que aún no gozan de los beneficios culturales y prácticos de la investigación científica. Más aún, la miseria de los países desarrollados podría ofrecer una oportunidad a los demás (...)" (BUNGE, 1982: 147). Ahondando aún más en la miseria del cientifismo técnico y sus características de desarrollo o socialización, Bunge trata el tema de la corrupción científica y del escaso índice de coherencia entre la realidad del ser humano, sus necesidades y la producción científica. Al igual que el desarrollo debe enfocarse globalmente, Bunge señala la necesidad de analizar la crisis científica como una parte más de esa globalidad de la que una parte fundamental es la propia imperfección o las carencias del sistema educativo: "(...) necesitamos, en suma, una participación más intensa y más racional de todos los interesados en el debate de la crisis global de la cual la crisis científica no es sino un aspecto (...)" (BUNGE, 1982: 154).

A este respecto García señala que se han 1legado a confundir lo que son los medios y los fines en la ciencia: "(...) poca gente parece cuestionar que el progreso de la ciencia sea una meta en sí misma, y pocas dudas cabe de que en la práctica lo es. Pero desde el planteamiento de que el ser humano debe controlar las técnicas que desarrolla y no viceversa, lo lógico sería que no fuese más que un instrumento para alcanzar los objetivos que sean directa 0 indirectamente vitales para el hombre (...)" (GARCÍA, 1992: 77).

En esta misma línea se manifiesta Habermas, para quien la racionalidad tecnológica reduce la acción práctica a una mera actividad ins- trumental y al análisis de los medios apropiados para determinados fines, olvidando el carácter específico e insoslayable del problema moral y político de los fines en toda actuación profesional que pretenda resolver problemas humanos (HABERMAS, 1984). Es por ello que el proceso de desarrollo no será realmente válido para su objeto - el ser humano en transformación optimizadora- hasta que no se supere la fase tecnológico científica y se llegue a afrontar desde los presupuestos de la educación crítica, participativa y transformadora de la realidad (emancipatoria).

La cuarta fase se corresponde con el concepto actual de desarrollo que se ha afianzado en su principal característica: la globalidad. Pero el carácter global del proceso de desarrollo va acompañado de la necesidad de transformar la realidad. Es, pues, un concepto normativo (no meramente descriptivo). La redistribución de la riqueza, la implicación de la población en la resolución de sus problemas, y la necesidad de educar al conjunto de la ciudadanía constituyen la piedra angular del concepto en cuestión.

En esta cuarta fase la educación se caracteriza por una dinamización de la transnacionalidad y la extensión del nivel secundario a un mayor sector de la población de los países en vías de desarrollo.

Ander-Egg aporta una visión integral del concepto de desarrollo muy en consonancia con el carácter global del mismo: "(...) sólo por comodidad metodológica o en un sentido muy parcial puede hablarse de desarrollo económico, político, cultural y social (...) hay que plantearlo como la búsqueda de una concepción integral o enfoque unificado del desarrollo (...)" (ANDER EGG, 1980). Este mismo autor señala que ya a finales de los cincuenta este enfoque integral del desarrollo fue tratado por el francés Lebret (perteneciente a la Escuela francesa de Economía y Humanismo), que se caracterizaba por concebir un desarrollo integral y armónico. En consonancia con su enfoque humanista a Lebret le preocupaba la obsesión por centrarse en la acumulación de recursos materiales: "(...) aprisionados por una teoría del poseer y de la extensión de la posesión, cuando en realidad había que subordinarlo todo al más ser y elaborar una teoría y una praxis del mas ser que comprendiese la utilización civilizadora del poseer (...) 
la finalidad del desarrollo no se agota, pues, en que los hombres tengan más, sino en que sean más (...)" (ANDER EGG, 1980). Lebret dotó a la disciplina económica del soporte humanista necesario para enfocar el desarrollo de forma armónica, integrando las ciencias sociales y explicitando la naturaleza teórico práxica de todo proceso de desarrollo. Por último, las Naciones Unidas enfocaron el tema del desarrollo de forma unificada lo que constituyó la universalización a nivel institucional de la educación para el desarrollo.

\section{DESARROLLO, COMUNIDAD Y ENFERMERÍA}

El concepto de desarrollo, que ya de por sí es complejo, acontece en un ámbito espacial cuya diversidad aumenta la variabilidad del proceso. Lo cierto es que no se puede hablar de desarrollo en abstracto, sino en el contexto local o comunitario, es decir en su caldo de cultivo. Ander-Egg entiende el desarrollo comunitario como el que: "(...) tiene por meta el que los grupos de las áreas, estructurados entre sí, sean aptos para encontrar las respuestas eficaces y obtengan la solución a los problemas que se presentan en la consecución de los valores que se han propuesto. Su desarrollo se logra por la acción comunitaria misma, concientiza a los grupos de su papel de gestores de una acción de desarrollo al nivel o escala en que ello es posible porque se sienten directamente comprometidos (...)" (ANDER-EGG, 1974). Ander Egg insiste en la necesidad de que sean los propios grupos integrantes de la comunidad los que participen activamente en el proceso de cambio de su realidad, aunque reconoce la complejidad de las interrelaciones que convergen en toda innovación (la comunidad tampoco puede tomar decisiones disarmónicas con el resto de las comunidades). El quid estriba en consagrar la dirección de la dinámica: "(...) desde la base a la cúspide y en una dimensión territorial, que tiende al encuentro con los planes globales y sectoriales de la cúspide a la base, fundamentalmente, permitiendo racionalizar los diversos espacios geográficos al posibilitar la creación de un tejido en el cual las áreas locales, zonales y regionales se integran y coordinan en función de recursos humanos, potencialidades naturales e interacciones sociales (...)" (ANDER-EGG, 1974). García aporta una definición del concepto de desa- rrollo desde el ámbito local entendiendo la entidad local, no como mero ejecutor de planes y controlador de procesos; las entidades locales deben transformarse en ejes dinamizadores de procesos de desarrollo: "(...) Si todo proceso de desarrollo constituye (o debe constituir) un intento de incidir en la mejora de los niveles de vida de la población de un territorio determinado, en el caso del desarrollo local este intento ha comportado una triple vertiente (...) dimensión económica (...) dimensión sociocultural (...) dimensión político-administrativa (...)" GARCÍA, 1992). Corroborando la aseveración de García pensamos que los municipios constituyen la institución idónea para diagnosticar los problemas de su propio contexto debido a su situación privilegiada de red local en la captación de problemas.

Ander Egg, después de afirmar que el término comunidad se refiere a una amplísima gama de realidades (un grupo de amigos, el barrio, el municipio, la provincia, la comunidad autónoma, la nación, la comunidad europea, etc), define el concepto de comunidad como una: "(...) agrupación organizada de personas que se perciben como una unidad social (...) cuyos miembros participan de algún rasgo, elemento, interés, objetivo o función; con conciencia de pertenencia y situados en una determinada área geográfica (...) en la cual la pluralidad de personas interacciona más intensamente entre sí que en otro contexto (...)" (ANDER EGG, 1980).

Para la existencia real de la enfermería comunitaria es preciso que la comunidad se convierta en dinamizadora del trabajo de enfermería. Sin duda, esta implicación de la comunidad en el diagnóstico y proceso de superación de sus problemas incluye, con carácter prioritario, el diagnóstico y proceso de superación de sus problemas de salud y, por la propia definición y naturaleza de Enfermería Comunitaria, hace imposible la existencia ninguna variedad -ni lejana ni aproximadade enfermería Comunitaria que no parta de este principio básico e ineluctable de IMPLICACIÓN de la comunidad. En consecuencia, difícilmente, podrá existir la enfermería comunitaria en países donde no imperen los principios, usos y prácticas democráticas (SILES, 1999).

La vinculación entre los términos comunidad, desarrollo y enfermería es evidente, puesto 
que toda planificación de desarrollo debe tener lugar en unidades sociales que tienen una ubicación geográfica específica. En una obra clásica del desarrollo de la comunidad Ross define este concepto como: "(...) un proceso por el cual una comunidad identifica sus necesidades en objetivos, ordena (o clasifica) otras necesidades en objetivos, halla los recursos (internos o externos), para enfrentarse con estas necesidades o alcanzar sus objetivos, actúa con respecto a los mismos y al hacerlo así se desarrollan en la comunidad actitudes cooperadoras y colaboradoras, y formas de actuar (...)" (ANDER EGG, 1980: 63). Ander Egg señala que el concepto de desarrollo de la comunidad depende, en última instancia del marco ideológico político desde el que se propicia y realiza el programa.

Pero además de la contextualización histórica mediante la que se contemplan las características de la comunidad y su evolución previa, no hay que caer en el fetichismo metodológico y aplicar el método de forma inflexible, puesto que si la tarea principal consiste en mejorar el ser humano, hay que ser coherentes y dejar un espacio a la creatividad que se ha de manifestar: "(...) en la imaginación sociológica para la captación de la realidad (...)" (ANDER-EGG, 1980: 99).

\section{ANÁLISIS CRÍTICO}

El concepto de desarrollo supera el nivel de objetivos meramente económicos, dado que dicho proceso debe afectar a los sistemas político y educativo, a la estructura social, sanitaria, y, por supuesto, al entramado económico del país en cuestión.

La perspectiva histórica resulta ser el recurso que mejor contribuye a profundizar en la crítica del concepto de desarrollo. ¿Donde se origina un término, tan aparentemente carente de rasgos de insolidaridad? Según Barraclouhg, los países ricos invirtieron enormes capitales en los países pobres (neocolonización o imperialismo capitalista), fundamentalmente la plusvalía estaba asegurada con las inversiones realizadas en los recursos naturales (BARRACLOUGH, 1979). Es decir, en estos países no era preciso que existiera un tejido industrial capaz de procesar sus propias materias (todavía no era rentable invertir en fábricas, puesto que estas estaban ubicadas en las propias metrópolis). En esta primera fase no era necesaria la educación de la población, tan sólo se precisaba una élite minoritaria y comprometida en el proceso de esquilmación de sus propios países.

Tras la I Guerra Mundial se pasó de la explotación de los recursos naturales a la necesidad de aprovechar al máximo las posibilidades existentes y a la creación de nuevas riquezas. La inversión este período se dirigió a los sectores más inmediatamente rentables para absorber los beneficios obtenidos sin política alguna de reinversiones. En esta fase se potenció el desarrollo dependiente de industrias selectivas (de inversiones extraordinariamente rentables a corto y medio plazo) para las que era precisa la existencia de mano de obra cualificada. En consecuencia, la educación se hizo necesaria para habilitar la mano de obra para las nuevas necesidades protoindustriales. En este marco, la salud de los obreros y los sistemas sanitarios responsables de la misma, no seguían criterios muy alejados de este enfoque marcadamente economicista y, en consecuencia, la enfermería no rebasó los límites de la aplicación de técnicas de forma rutinaria ocupando la humilde, pasiva y hasta servil posición que le correspondía en la escala burocrática del sistema sanitario. Tras la II Guerra Mundial se evidenció de una forma tal el dualismo económico y social que la dinámica de bloques se lanzó de lleno a aumentar sus zonas de influencia utilizando para ello la política de desarrollo con su correspondiente apéndice educacional.

-El fracaso de la política de desarrollo generada en los años cincuenta fue calificada por Sampedro como "patologías de la cultura industrial" centrada en un enfoque exclusivamente económico (patología economicista). La perspectiva cuantitativista, neopositivista partía de la premisa de que el crecimiento económico provocaría el impulso del resto de las condiciones de la población: sociales, culturales, políticas. Este enfoque adolecía del denominado "fetichismo del crecimiento" (GARCÍA, 1992: 76).

En definitiva el holismo que comparten la educación para el desarrollo y la enfermería transcultural lo que exige es, fundamentalmente, desarrollar una capacidad de interpretación interdisciplinaria de la realidad, ocupándose la educación para el desarrollo de acotar una perspectiva genérica de la cuestión mientras que la enfermería trans- 
cultural se centraría en una parte más específica de la misma: los cuidados transculturales. La gran dificultad que esta empresa comporta estriba en la costumbre, institucionalizada por la comunidad científica, de acotar los problemas concretos desde parcelas aisladas: "En lugar de una aproximación especializada que separe los problemas económicos, sociales y medioambientales, es preciso reflexionar de forma global, sistémica, una reflexión para la cual nuestra educación y nuestras formaciones apenas nos han preparado(...)" (CELORIO, 1999).

\section{EL PAPEL DE LA ENFERMERÍA EN EDUCACIÓN PARA EL DESARROLLO}

La esencia de la enfermería y la antropología son consustanciales con la propia naturaleza de la educación para el desarrollo (LEININGER, 1984). Esto es así porque si la enfermería y la antropología se ocupan de estudiar los mecanismos que la comunidad emplea en la satisfacción de sus necesidades, toda la teoría y la práctica de la educación para el desarrollo gira en torno a la necesidad de mejorar ese mecanismo de satisfacción de necesidades. La enfermería transcultural se ocupa, además, de estudiar las diferencias culturales que se dan en ese proceso de satisfacción de necesidades (LEININGER, 1985) con el fin de hacer una enfermería más eficaz mediante la tolerancia, el respeto intercultural y, en fin, la armonización transcultural

\section{AGENTES TRANSVERSALES EN EDUCACIÓN PARA EL DESARROLLO Y ENFERMERÍA TRANSCULTURAL: EL FORO DE ENFERMERÍA PARA EL DESARROLLO}

En palabras de Celorio (1999), los agentes transversales son aquellos individuos, colectivos civiles, plataformas sociales, etc. que, desde dentro o fuera del sistema educativo y/o sanitario, buscan fórmulas para llevar a la práctica el compromiso de cambio con el que desde sus diferentes campos y especialidades se han comprometido. La transformación práctica de las situaciones sociales, políticas y económicas constituyen un gran frente de trabajo que debe complementarse con la transformación de las situaciones de salud-enfermedad. El primer paso en este proceso consiste en la toma de conciencia histórica del rechazo que toda iniciativa de construcción innovadora va a provocar en el poder reproductivo del modelo dominante que anida en todo sistema cultural, lo cual implicará una puesta en escena del componente ideológico - tan importante como ignorado en el contexto científico y educativo - y facilitará la fundamentación de un compromiso ideológico en materia educativa y sociosanitaria. Para llegar a este punto es preciso activar la capacidad crítica de la sociedad y, especialmente, de educadores y enfermeros, aplicándola al rechazo de lo injusto e indigno dentro de los sistemas sanitarios y educativos $\mathrm{y}$, asimismo, al diseño de logros deseables en culturas concretas. Pero para que todo este proceso se pueda fundamentar en un contexto científico, es preciso desarrollar una profunda reflexión epistemológica que fundamente el papel innovador del aprendizaje a nivel educativo y sociosanitario orientando el proceso de enseñanza-aprendizaje hacia situaciones prácticas de cambio cultural (TABLA I). La actividad en el aula puede formar constituir una acción de educación para el desarrollo si se incluyen en los currículos los contenidos necesarios para favorecer el proceso de concientización en un clima de enseñanza-aprendizaje lo suficientemente adecuado. Una consecuencia práctica de estas actividades de educación para el desarrollo en el aula es la formación de asociaciones y ONG como el Foro de Enfermería para el Desarrollo fundado a mediados de los noventa partiendo de un grupo de alumnos aglutinado en torno a una asignatura "Pedagogía aplicada a los cuidados" en la E.U. de Enfermería de Alicante.

\section{Tabla I}

a) Fundamentar lo educativo y lo sociosanitario en un Compromiso Ideológico:

- En sistemas de valores alternativos en materia educativa y situaciones de salud-enfermedad

- En sectores sociales y sanitarios a los que se considera sujetos de su acción

- En alternativas emancipadoras de organización social y sanitaria

- En fomentar la implicación orientada a la acción

- En la acción organizada y su participación

b) Implicar algunas formas de Análisis Crítico de 
la Sociedad:

- En crítica y negación de las formas y efectos del presente dominante

- En afirmación de formas de futuro tenidas como deseables,

- En líneas y estrategias del cambio y transformación social,

- En los recursos necesarios para que los sujetos a quienes liga el propio compromiso transformen su situación

c) Desarrollar una epistemología implícita sobre la Relación Conocimiento/Praxis

- En nuevas formas de construir y organizar el conocimiento relativo a educación y situaciones de salud-enfermedad

- En los supuestos ideológicos y valorativos.

- En formas de socialización para generar espacios sociales de cambio

- En estilos genuinos para situar el conocimiento en la lógica de la acción

- En el sentido del conocimiento en la práctica organizada

d) Conceder potencialidad al Aprendizaje para la Innovación Social

- En la capacidad de ser herramienta para sujetos y colectivos

- En la capacidad de construir conciencia crítica ante la realidad

- En la capacidad de ayudar a reelaborar sistemas de valores

- En la capacidad de captar lo nuevo al tiempo que se construye en la práctica

- En la capacidad de ser instrumento de intercambio creativo

e) Entender e impulsar la Acción educativa y sociosanitaria como Acción para el Cambio

- No interesa como acción con sentido académico

- Nunca es una acción socialmente neutral

- Se construye social y organizadamente lo que en si mismo educa

- La educación es una acción inevitablemente transformadora

- Interactúa con otras fuerzas que "educan" también y en paralelo

- Pretende producir cambios en determinada dirección

- Forma parte de la lógica de las estrategias de la acción organizada

- Implica éticamente a los sujetos que entran en la interacción educativa

- Conciencia crítica ante la educación reproductiva del modelo

Fuente: CELORIO, J. (1999) De la ED como Transversal a la Globalización Crítica y Alternativa de la Renovación Educativa. Publicaciones Universidad del Pais Vasco, Bilbao

\section{MARCO CIENTÍFICO DE LA EDUCACIÓN PARA EL DESARROLLO Y LA ENFERMERÍA TRANSCULTURAL}

Siguiendo a Kuhn (1995), se puede interpretar el concepto de paradigma como el soporte referencial que sirve para aglutinar los ejemplares compartidos de una rama particular de la ciencia, un colegio invisible o sistema de citas mutuas y, también, el sistema de referencias que sirve para esgrimir criterios de aceptación y rechazo de los trabajos que deben emprenderse, subvencionarse o publicarse; $y$, por último, se puede interpretar la función de los paradigmas como aquel sistema que sirve para dotar de modelos de problemas y soluciones a la comunidad científica (SILES y GARCÍA, 1995; SILES, 1996, 1997). Desde esta perspectiva, tanto la educación para el desarrollo como la enfermería antropológica y transcultural no son aceptadas de forma genuina y sin ambajes más que por un paradigma de entre las tres plataformas o sistemas de referencias científicas universalmente reconocidos en la actualidad: el paradigma sociocrítico. Esta realidad debe entenderse desde el conocimiento y análisis concienzudo de las características de los paradigmas racional tecnológico, hermenéutico y sociocrítico (GONZÁLEZ HERNÁNDEZ, SILES, 1996, 1997) (Tablas II, III y IV).

\section{Tabla III}

EDUCACIÓN PARA EL DESARROLLO Y ENFERMERÍA ANTROPOLÓGICA Y TRANSCULTURAL EN EL PARADIGMA RACIONAL TECNOLÓGICO

\section{E-D}

\section{EDUCADOR}

-AGENTE CONTROLADOR DEL PROCESO 
DE E-D.

-MANIPULADOR EXTERNO Y OBJETIVO RESPECTO DE LAS NECESIDADES FORMATIVAS DE LAS CULTURAS TRATADAS.

-APLICADOR DE PRESCRIPCIONES Y TÉCNICAS PEDAGÓGICAS .

-ADMINISTRADOR TÉCNICO DEL PROCESO DE E-D.

\section{EDUCANDO (CULTURAS ESTUDIADAS)}

-OBJETO DE CONTROL Y DESTINATARIO DE PRESCRIPCIONES EDUCATIVAS.

-ACATAMIENTO PASIVO DE NORMAS, PRESCRIPCIONES Y PROCEDIMIENTOS DE E-D.

\section{CONCEPTO DE E-D}

-CONCEPTO NEOPOSITIVISTA: E-D COMO MECANISMO DE TRANSMISIÓN DE NUEVAS TECNOLOGÍAS Y RUTINAS ESTANDARIZAS DE PROCESOS EXTERNOS A LAS CULTURAS TRATADAS.

\section{TIPO DE CONOCIMIENTO CONSTRUIDO}

-EDUCACIÓN COMO CIENCIA NATURAL HEREDERA DEL NEOPOSITIVISMO

-CARÁCTER OBJETIVO DE LA ACTIVIDAD CIENTÍFICA Y EDUCATIVA

-CARÁCTER OBJETIVO DE LAS ACCIONES DE E-D

-PREEMINENCIA DE ACCIONES DE CUANTIFICACIÓN-NEUTRALIDAD.

-CONTROL EXTERNO DEL PROCESO E-D.

\section{¿CÓMO SE CONSTRUYE?}

-INVESTIGACIÓN EXPERIMENTAL (PRUEBAS ESTANDARIZADAS, TEST, ENCUESTAS).

-GRAN PROTAGONISMO DE LA INSTRUMENTACIÓN ESTADÍSTICA.

-PROCESO CIENTÍFICO Y DE E-D AJENO Y SUPERIOR AL OBJETO-SUJETO (CULTURAS).

\section{¿PARA QUÉ SIRVE?}

-LA TEORÍA DIRIGE LA ACCIÓN SEPARADA DE LA PRAXIS.

-LA PRÁCTICA SE MODIFICA TEÓRICAMENTE.

-LA FORMACIÓN SE ENFOCA COMO UN
PROCESO TECNOLÓGICO.

-FORMADOR COMO EJECUTOR DE TÉCNICAS.

\section{$E^{a}$ ANTROPOLÓGICA Y $\mathbb{E}^{\mathrm{a}}$ TRANSCULTURAL}

CON LOS PRESUPUESTOS DEL PARADIGMA RACIONAL TECNOLÓGICO NO SE PUEDE COMPRENDER NI LA E E $^{\mathrm{a}}$ ANTROPOLÓGICA, NI TAMPOCO LA ENFERMERÍA TRANSCULTURAL

Fuente: Siles, J. (1997) Epistemología y Enfermeria: por una fundamentación cientifica y profesional de la disciplina. Enfermeria Clínica. 7/4: 38-44

\section{Tabla III}

\section{EDUCACIÓN PARA EL DESARROLLO Y ENFERMERÍA ANTROPOLÓGICA Y TRANSCULTURAL EN EL PARADIGMA HERMENÉUTICO}

\section{E-D}

\section{EDUCADOR}

-AGENTE DE COMUNICACIÓN COMPRENSIVA E INTERACTIVA.

-INTERPRETADOR DE LA REALIDAD DEL FORMANDO

CULTURA ESTUDIADA

-SUJETO COMUNICADOR DE SU ESTADO Y SUS EXPECTATIVAS (INTERPRETACIÓN DE SU SITUACIÓN SOCIOECONÓMICA, POLÍTICA, SANITARIA Y FORMATIVA).

CONCEPTO DE E-D

-CONCEPTO SITUACIONAL ELABORADO INTERACTIVAMENTE .

\section{TIPO DE CONOCIMIENTO CONSTRUIDO}

-E-D COMO CONSTRUCCIÓN PERSONAL -SOCIOLOGÍA INTERACCIONISTA.

-HERMENÉUTICA Y FENOMENOLOGÍA EDUCATIVA-ANTROPOLOGÍA CULTURAL.

\section{¿CÓMO SE CONSTRUYE?}

-INVESTIGACIÓN ETNOGRÁFICA. -ESTUDIO DE SIGNIFICADOS EN SUS CON- 
TEXTOS (USO DE MÉTODOS Y TÉCNICAS CUALITATIVAS, NARRATIVAS, MATERIAL BIOGRÁFICO Y AUTOBIOGRÁFICO)

-OBSERVACIÓN PARTICIPANTE, NOTAS DE CAMPO, DIARIOS, RELATOS, ETC.

\section{¿PARA QUÉ SIRVE?}

-LA PRÁCTICA ES EL FUNDAMENTO DE LA TEORÍA (SU PRESUPUESTO)

-E-D ENFOCADA COMO PROCESO DE INTERCOMUNICACIÓN FORMADOR-FORMANDO.

-INTERVENCIÓN PRÁCTICA INTERPRETATIVA. ACTO DE COMPRENSIÓN DE SITUACIONES EDUCATIVAS.

\section{$E^{\text {a }}$ ANTROPOLÓGICA}

\section{ENFERMERO-A}

-AGENTE DE COMUNICACIÓN COMPRENSIVA E INTERACTIVA.

-INTERPRETADOR DE LA REALIDAD DEL PACIENTE INMERSO EN CU SU CULTURA

\section{CULTURA ESTUDIADA}

-SUJETO COMUNICADOR DE SU ESTADO Y SUS EXPECTATIVAS (INTERPRETACIÓN DE SU SITUACIÓN DE SALUD).

\section{CONCEPTO DE E ANTROPOLÓGICA}

-CONCEPTO SITUACIONAL ELABORADO INTERACTIVAMENTE .

\section{TIPO DE CONOCIMIENTO CONSTRUIDO}

-EaATROPOLÓGICA Y TRANSCULTURAL COMO CONSTRUCCIÓN PERSONAL -SOCIOLOGÍA INTERACCIONISTA. -HERMENÉUTICA Y FENOMENOLOGÍA EDUCATIVA-ANTROPOLOGÍA CULTURAL.

¿CÓMO SE CONSTRUYE?

-INVESTIGACIÓN ETNOGRÁFICA. -ESTUDIO DE SIGNIFICADOS EN SUS CONTEXTOS (USO DE MÉTODOS Y TÉCNICAS CUALITATIVAS, NARRATIVAS, MATERIAL BIOGRÁFICO Y AUTOBIOGRÁFICO) -OBSERVACIÓN PARTICIPANTE, NOTAS DE CAMPO, DIARIOS, RELATOS, ETC.
TEORÍA (SU PRESUPUESTO)

- E $^{\mathrm{a}}$ ANTROPOLÓGICA ENFOCADA COMO PROCESO DE INTERCOMUNICACIÓN CULTURA TRATADA(INDIVIDUO, FAMILIA, COMUNIDAD/ ENFERMERO-A..

-INTERVENCIÓN PRÁCTICA INTERPRETATIVA. ACTO DE COMPRENSIÓN DE SITUACIONES DE SALUD-ENFERMEDAD.

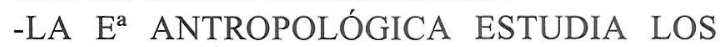
MECANISMOS DE SATISFACCIÓN DE NECESIDADES RELATIVAS A SITUACIONES DE SALUD-ENFERMEDAD DE UNA DETERMINADA CULTURA Y CONSTITUYE EL PASO PREVIO PARA EL ANÁLISIS COMPARATIVO INTERCULTURAL QUE SE ESTABLECE EN LA E ${ }^{\text {a }}$ TRANSCULTURAL.

Fuente: Siles, J. (1997) Epistemologia y Enfermeria: por una fundamentación científica y profesional de la disciplina. Enfermería Clínica. 7/4: 38-44

\section{Tabla IV}

\section{EDUCACIÓN PARA EL DESARROLLO Y ENFERMERÍA ANTROPOLÓGICA Y TRANSCULTURAL EN EL PARADIGMA SOCIOCRÍTICO}

\section{E-D}

\section{EDUCADOR}

-AGENTE DE CAMBIO CULTURAL: SOCIAL, SANITARIO, EDUCATIVO, POLÍTICO Y ECONÓMICO.

\section{CULTURA ESTUDIADA}

-IMPLICADA EN SU PROPIO PROCESO DE CAMBIO SOCIAL, SANITARIO, EDUCATIVO, POLÍTICO Y ECONÓMICO.

\section{CONCEPTO DE E-D}

-CONCEPTO POSITIVO.

-REELABORACIÓN DEL MISMO POR PARTE DE LA CULTURA OBJETO-SUJETO DE ESTUDIO.

\section{TIPO DE CONOCIMIENTO CONSTRUIDO} -E-D COMO CONSTRUCCIÓN CULTURAL -DIALÉCTICA-SUBJETIVIDAD 
-CONSTRUCCIÓN NUEVAS REALIDADES SOCIALES, SANITARIAS, EDUCATIVAS, POLÍTICAS Y ECONÓMICAS (INNOVACIONES).

\section{¿CÓMO SE CONSTRUYE?}

-INVESTIGACIÓN CRÍTICA

-INVESTIGACIÓN ACCIÓN

-ESTUDIOS DE CONTEXTOS PERSONALES Y

SOCIALES.

-COMPROMISO PARA SOLUCIÓN DE PROBLEMAS.

-LAS CULTURAS Y LOS SUJETOS QUE LAS
CONFIGURAN POR ENCIMA DE LOS
MÉTODOS.

\section{¿PARA QUÉ SIRVE?}

-LA PRÁCTICA SE MODIFICA TEÓRICA Y PRÁCTICAMENTE

-LA E-D ENFOCADA COMO INTERVENCIÓN EN PRÁCTICA SOCIAL Y ANTROPOLÓGICA DEL SUJETO.

-EL PROCESO DE TOMA DE DECISIONES CENTRADO EN LA CAPACIDAD CRÍTICA.

\section{$\mathrm{E}^{\mathrm{a}}$ ANTROPOLÓGICA}

ENFERMERO-A: AGENTE DE COMUNICACIÓN COMPRENSIVA COMO PASO PREVIO E INELUDIBLE DE LA E ${ }^{\mathrm{a}}$ TRANSCULTURAL.

\section{ENFERMERÍA TRANSCULTURAL}

ENFERMERO-A: AGENTE DE CAMBIO SOCIOSANITARIO QUE IMPLICA PARTICIPATIVAMENTE AL INDIVIDUO, FAMILIA Y COMUNIDAD EN EL CONTROL DE SUS SITUACIONES DE SALUD-ENFERMEDAD Y, ESPECIALMENTE, EN LA ARMONIZACIÓN DE LAS DIFERENCIAS CULTURALES PROPIAS DE DICHAS SITUACIONES.

Fuente: Siles, J. (1997) Epistemología y Enfermería: por una fundamentación cientifica y profesional de la disciplina. Enfermería Clínica. 7/4: 38-44

\section{PERTINENCIA DE LA EDUCACIÓN PARA EL DESARROLLO Y LA ENFERMERÍA TRANSCULTURAL}

Los últimos análisis de las tendencias migratorias en diversos países de la Comunidad Europea
(OCDE, 1998), y en España (PAJARES, 1998; INSTITUTO DE ESTUDIOS TRANSNACIONALES, 1997); alertan sobre la necesidad de desarrollar políticas adecuadas para hacer frente a las nuevas situaciones, de forma integral, de colectivos de inmigrantes que van a necesitar un importante apoyo educativo y sociosanitario en el que la educación para el desarrollo y la enfermería transcultural deben desempeñar un papel de primer orden. Uno de los problemas que acechan de forma especialmente preocupante en este contexto inmigratorio es el racismo de forma que se deben activar las conciencias para no acabar practicando una enfermería parcelada por razas (blancos occidentales, blancos del este, negros, musulmanes, gitanos, etc.) (BARBADILLO GRIÑÁN, 1997; CALVO BUEZAS, 1997; ESPADA, M².L. 1997; VV.AA. 1997). Pero también resulta preocupante otro tipo de marginación potencial relacionado con la migración femenina y la peligrosa mezcolanza de elementos religiosos y de género (GREGORIO, 1998; RAMÍREZ, 1998). Sin duda alguna la sociedad española - y también la europea-, deben prepararse para una convivencia plural. Pero la convivencia multirracial implica cuidados transculturales (KRISTEVA, 1991) y para desarrollar una enfermería comunitaria y transcultural sin duda es preciso ir más allá de las investigaciones teóricas y desarrollar un soporte normativo e institucional acorde con las necesidades generadas por los flujos de población (KYMLICKA, 1995), por lo que los procesos de concienciación deben llegar a impregnar la clase política y las élites burocráticas. Una de las estrategias a desarrollar es marcar un objetivo común a todos los que conviven en una misma sociedad desde perspectivas culturales diferentes: la convivencia en tolerancia y respeto. Todo este proceso se puede sintetizar con la necesidad de conseguir un clima de tolerancia y solidaridad que haga posible la convivencia, es decir, un ambiente de paz (SEMINARIO DE INVESTIGACIÓN PARA LA PAZ , 1996.

\section{LOS TRES MOMENTOS CULTURALES Y EL PAPEL DE LA ENFERMERÍA}

En el terreno de la enfermería, para que se de en la práctica ese respeto reclamado hacia todos los colectivos que integran la comunidad plural del 
siglo XXI, es preciso comprender que es imposible respetar sin conocer. Dicho de otra forma, para que se pueda practicar con expectativas de eficacia la enfermería transcultural, debe profundizarse en el conocimiento de esa comunidad plural; esto es, los enfermeros deben estudiar antropológicamente las características de todos y cada uno de los colectivos que configuran la pluralidad de la comunidad. No sólo existe una enfermería transcultural, sino que más bien, este tipo de enfermería holística y armonizadora es el ideal u objetivo a alcanzar en una sociedad plural. En una primera fase se puede hablar de la existencia de una enfermería de tipo multicultural cuya principal característica consiste en el aislamiento recíproco de las diversas culturas que componen una sociedad plural pero con compartimentos estancos, lo que implica un desconocimiento mutuo. En una segunda fase se producen los primeros contactos e intentan establecerse relaciones e intercambios mediante los que se puede generar el conocimiento y el respeto mutuo. A este tipo de enfermería se la denomina enfermería intercultural. Por último, cuando se armonizan las diferencias y se practica una enfermería culturalmente sintética orientada fundamentalmente por el respeto y la implicación activa de los pacientes, se puede hablar, por fin, de la existencia de una enfermería transcultural (FIGURA I).

Para llegar al conocimiento de la comunidad plural, necesario para la práctica enfermera, se propone un proceso de investigación-acción flexible que se adapte a las peculiaridades del contexto investigado (TABLA V). En la fase preliminar el proceso de selección de métodos y técnicas hunde sus raíces en el mismo concepto de cultura del que se parte, por lo que resulta tan clarificador como conveniente reflejar de forma concisa el mismo. Desde esta línea investigadora la definición resulta apropiada la definición de Malinowski (1984) que concibe la cultura como la totalidad de los comportamientos que una comunidad orienta con respecto a un fin: la satisfacción del sistema de necesidades. Desde esta perspectiva, la antropología se ocupa del estudio de los diferentes comportamientos implicados en el proceso de satisfacción de necesidades de la comunidad y también de los componentes ideológicos, míticos y religiosos que le dan un sentido a dicho proceso. El estudio de los mecanismos de satisfacción de necesidades emple- ados por las estructuras sociales resulta tan eficaz para la antropología como para la enfermería transcultural y para la propia enfermería comunitaria . Por tanto, en primer lugar, se ha de partir de un estudio histórico-antropológico mediante el que se procederá al diagnóstico de los principales problemas y a la identificación de las concomitantes culturales derivadas del proceso de satisfacción de necesidades. En una segunda fase se procede al diseño del programa educativo. Posteriormente se implementa dicho programa y, finalmente, se procede a su evaluación

\section{Tabla V}

\section{- INVESTIGACIÓN HISTÓRICO-ANTROPO- LÓGICA \\ -DIAGNÓSTICO PRINCIPALES PROBLE- MAS}

-IDENTIFICACIÓN DE LAS CONCOMITANCIAS CULTURALES DERIVADAS DEL PROCESO DE SATISFACCIÓN DE NECESIDADES

- DISEÑO PROGRAMA EDUCATIVO

- APLICACIÓN DEL PROGRAMA EDUCATIVO - EVALUACIÓN

\section{CONCLUSIONES}

-Existe una estrecha vinculación entre el concepto concreto de "desarrollo" y la visión particular sobre el tema educativo. Este vínculo debe orientar de forma concisa la adopción de procedimientos y métodos de investigación-educación.

-Los métodos y técnicas de investigación acción resultan más adecuados en educación para el desarrollo, dado que sus fines no se agotan en la producción de conocimientos, sino que se considera la necesidad de emplear los resultados de la realidad estudiada en el mejoramiento de la misma. No se agota el proceso científico en la producción de conocimientos.

-En enfermería existen oenegés que han tomado conciencia de esta problemática (Foro de Enfermería para el Desarrollo)

-El concepto de desarrollo surge cuando se hace preciso adaptar la estructura socioeconómica de las antiguas colonias y zonas de influencia a las nuevas necesidades del capitalismo tras la II 


\section{Guerra Mundial.}

-La educación ha sido utilizada como instrumento para alcanzar los fines del desarrollismo. Según el nivel de globalidad y participación de la población y la democratización del sistema político, la organización y la planificación del desarrollo ha orientado el papel de la educación desde la perspectiva tecnológica (formadora de técnicos ¿tan neutrales como el propio sistema educativo?), hasta la crítica (implicación activa de la población en la transformación de su propia realidad).

-El concepto de desarrollo y la necesidad de aplicar sus principios, surge tras la II Guerra Mundial. La posguerra estuvo caracterizada por el inicio de la bipolarización política y económica y las iniciativas acometidas por el bloque socialista difirieron sustancialmente de las del otro bloque. En definitiva y aun mucho antes del sistema bipolar de bloques, el concepto de desarrollo trasciende el ámbito meramente nacional, y esto es un hecho constatado por los principales investigadores y actores de la política internacional.

-El desarrollo y el subdesarrollo constituyen las dos caras de la misma moneda. La historia de los países desarrollados muestra nítidamente cómo y a costa de qué y de quienes alcanzaron el desarrollo en un contexto económico y político que les favorecía. La historia, así mismo, explica en que medida el desarrollo de los países ricos truncó sus posibilidades de expansión autónoma, explicitando las causas de su actual nivel de dependencia en el orden económico y político.

-Desde la óptica bipolar, el desarrollismo fue utilizado por el bloque capitalista para frenar las reivindicaciones políticas y sociales de los sectores más deprimidos de los países pobres.

-El núcleo dirigente de los países "adoptados" en los planes de desarrollo del bloque capitalista sirvió en todo momento los intereses de la potencia protectora de sus privilegiadas situaciones. Los intereses de la nación quedaban, obviamente, relegados a planos microscópicos.

-Se evidencia la evolución histórica y, hasta cierto punto paralela, de los conceptos "desarrollo" y "educación". En un principio la educación -en los años cincuenta, tras la II Guerra Mundial- es concebida como un instrumento fundamental para formar en habilidades y conocimientos a los nuevos técnicos de los países subdesarrollados.

-La multinacionales generan puestos de trabajo y riqueza siempre y cuando se cumplan una serie de requisitos políticos y económicos, pero contribuyen a aumentar el nivel de dependencia de de las naciones que dependen de ellas.

-Tras la revolución del 68 se alzaron voces contra lo que consideraban sólo era una forma de estabilizar el sistema capitalista.

-El desarrollo sustentado exclusivamente en criterios economicistas y tecnologicistas al ignorar las características del ser humano somete a éste a una suerte de tiranía a la que no cabe otra salida más que la adaptación. El humanismo debe primar sobre los criterios tecnológicos, dado que son éstos los que tienen que plegarse a las necesidades del hombre y no al revés.

-Cuando no existe voluntad de fomentar la participación crítica de la población como mediadores en el proceso de toma de decisiones de un sistema político sensible a los intereses generales, la inducción al desarrollo resulta artificiosa y oculta la verdadera finalidad del proceso: mantener el vínculo de dependencia con respecto a la antigua metrópoli.

-Es lícito afirmar que el concepto actual de desarrollo está marcado por su carácter global y por la dimensión teleológica respecto a la intervención de la realidad en sentido optimizador y humanizante. Siguiendo a Habermas, pensamos que la racionalidad tecnológica reduce la acción práctica a una mera actividad instrumental y al análisis de los medios apropiados para determinados fines, olvidando el carácter específico e insoslayable del problema moral y político de los fines en toda actuación profesional que pretenda resolver problemas humanos (HABERMAS, 1984). Es por ello que el proceso de desarrollo no será realmente válido para su objeto -el ser humano en transformación optimizadora- hasta que no se supere la fase tecnológico científica y se llegue a

-El concepto de desarrollo, que ya de por sí es complejo, acontece en un ámbito espacial cuya diversidad aumenta la variabilidad del proceso. Cualquier planificación de desarrollo debera tener en cuenta su contexto comunitario.

-Por la propia definición de Enfermería Comunitaria, resulta del todo imposible el desarro- 
llo de la enfermería comunitaria que no parta de los principios básicos que comparte con la educación para el desarrollo y la enfermería transcultural.

-Las carencias educativas y la crisis científica -o la ineficacia que padece como consecuencia de su alto nivel de corrupción- no son sino subconjuntos de la crisis global de la humanidad en la que coexisten la miseria y la injusticia conjuntamente con los medios tecnológicos y científicos para su erradicación.

-Esta crisis global sólo puede afrontarse mediante plantemientos que tengan en cuenta la naturaleza del ser humano, puesto que, además de ir dirigida al mismo, son los hombres los que deciden las políticas, las metodologías, las técnicas y estrategias a seguir.

-En la última etapa de la educación para el desarrollo, la etapa ecológica o armonizadora, el transculturalismo y el transnacionalismo constituyen dos nuevos planteamientos que afectan tanto a la enfermería como a la propia educación para el desarrollo.

-Sólo desde los presupuestos del paradigma sociocrítico se pueden desarrollar acciones innovadoras y emancipadoras propias de la Educación para el Desarrollo y la enfermería transcultural.

-Para poder realizar comprensivamente este trabajo ha sido preciso proceder a la clarificación explicita de conceptos asociados (política e ideología). La política y la ideología suponen una influencia de un calibre tal que sus significados deben ser analizados conjuntamente (política e ideología educativa, política e ideología del desarrollo o desarrollismo).

-Para que se pueda hablar de la existencia tanto de enfermería comunitaria como de enfermería transcultural es preciso implicar a la población transformandola en dinamizadora del proceso.

-Sólo en países en los que imperen los sistemas democráticos y se practiquen los usos y costumbres participativas es posible realizar plenamente la enfermería comunitaria y la enfermería transcultural.

-La principal diferencia entre la enfermería antropológica y la enfermería transcultural estriba en el hecho de que la primera estudia los mecanismos de satisfacción de necesidades y sus concomitnates culturales de una comunidad desde la perspectiva de las situaciones salud-enfermedad, mientras que la segunda analiza comparativamente las diferencias en los mecanismos de satisfacción de necesidades y sus concomitantes culturales de distintas comunidades con una finalidad práctica: armonizar esas diferencias mediante acciones de enfermería transculturales.

-Tanto, la Educación para el Desarrollo como la enfermería transcultural tienen enormes dificultades para desarrollarse en un contexto completamente hostil: racismo, xenofobia, guerra, etc.

-Los principios, métodos y prácticas de la educación para el desarrollo, la enfermería comunitaria y la enfermería transcultural están estrechamente vinculados dado que parten de los mismos presupuestos: implicación participativa y responsable de la comunidad en el proceso de satisfacción de sus necesidades. Sólo desde la perspectiva del paradigma sociocrítico se pueden desarrollar estas tres disciplinas, dado que es el único paradigma que propugna la implicación participativa y responsable de los sujetos investigados.

-Los métodos y técnicas propios de la investigación acción constituyen los más idóneos para la el diagnóstic, adopción e implementación de actividades en educación para el desarrollo, enfermería transcultural y enfermería comunitaria.

-La enfermería antropológica y transcultural, tanto por su esencia como por su carácter pedagógico y sociocrítico, constituye una pieza fundamental en todo lo relacionado con educación para el desarrollo.

\section{BIBLIOGAFÍA}

ALEMÁN BRACHO, M.C. (1991) El sistema público de servicios sociales en España. Impredisur, Granada.

ÁLVAREZ, J.J. (et al) (1989) Organizaciones voluntarias e intervención social. Acebo, Madrid.

ANDER-EGG, E. (1969) Introducción a las técnicas de investigación social. Humanitas, Buenos Aires.

-(1974) El trabajo social como acción liberadora. Ecro, Buenos Aires.

-(1980) Metodología y práctica del desarrollo de la comunidad. Unieuropa, Valencia.

-(1985) Ideología política y trabajo social. Humanitas, Buenos Aires.

BARBADILLO GRIÑÁN, P. (1997); Extranjería, 
racismo y xenofobia en la España contemporánea. La evolución de los setenta a los noventa, Madrid: Centro de Investigaciones Sociológicas/Siglo XXI de España editores, colección "monografías", 154.

BATTEN, T.R. (1965) Preparación para el desarrollo comunitario. Euroamérica, Madrid.

BONFLIGIO, G. (1982) Desarrollo de la comunidad y trabajo social. Gelats, Lima.

BORRAJO, E. (1966) Política social y servicios sociales. Doncel, Madrid.

BUNGE, m. (1982) Economía y Filosofía. Tecnos, Madrid

CALVO BUEZAS, T. (1997); Racismo y solidaridad de españoles, portugueses y latinoamericanos. Los jóvenes ante otros pueblos y culturas, Madrid: Ediciones Libertarias.

CASADO, D. (1977) Cambio de las instituciones de bienestar social en España. Marova, Madrid. -(1987) Introducción a los servicios sociales. Acebo, Madrid.

CELORIO, J. (1999) De la ED como Transversal a la Globalización Crítica y Alternativa de la renovación Educativa. Publicaciones Universidad del País Vasco, Bilbao

CORRIGAN, P. (1984) Social Work under capitalism: marxist approach. MaCmillan Publishers, London.

CRUZ ROCHE, I. (1984) Análisis de los costes de las instituciones sanitarias. En: Revista de la Seguridad Social, 22: 25-44.

DI CARLO, E. (1983) Necesidades básicas y cambio social. Humanitas, Buenos Aires.

ESPADA, Mª L. (1997); ¿Europa, ciudad abierta? La inmigración y el asilo en la Unión Europea, Granada: Instituto Municipal de Formación y Empleo.

FABEIROS, V. Trabajo social e instituciones. Humanitas, Buenos Aires.

GARCÍA, A. (1990) Ciencias sociales y epistemología. PPU, Barcelona.

-(1992) Ciencias sociales y epistemología: el caso de la pedagogía social. PPU, Barcelona GONZÁLEZ HERNÁNDEZ, A. (1989) La investigación acción como metodología en las ciencias sociales. Cossio, Murcia.

GREFFE, X. (1980) Partage du travail et mode de développement. Droit Social, 1: 86-99

GREFFE, X. (et al) (1982) L impot. Presses uni- versitaires de France, París.

GREGORIO, C. (1998); Migración femenina. Su impacto en las relaciones de género. Narcea, Madrid.

HABERMAS, J (1984) Ciencia y técnica como ideología. Tecnos, Madrid.

INSTITUTO DE ESTUDIOS TRANSNACIONALES, 1997); Mediterráneo, ¿camino abierto o frontera?, Córdoba: INET

INSTITUTO DE MIGRACIONES Y SERVICIOS SOCIALES (1998) Actitudes hacia los inmigrantes, Ministerio de Trabajo y Asuntos Sociales, Madrid.

KISNERMAN, N. (1984) Comunidad. Humanitas, Buenos Aires.

KRISTEVA, J. (1991); Extranjeros para nosotros mismos: ¿Será posible la convivencia multirracial en la Europa del siglo XX?. Plaza \& Janes, Barcelona

KUHN, T.S. (1995) ¿Qué son las revoluciones científicas? Ediciones Altaya, Barcelona.

KYMLICKA, W. (1995) The Rights of Minority Cultures. Oxford University Press, New York.

LANE, A.J. (1996) Developing healthcare educators: the application of a conceptual model. Journal-of-Nursing-Staff-development, 12/5: 252-9

LEININGER, M. (1984) Care: The essence of nursing and health. NJ Slack, Thorofare.

-(1985) Transcultural nursing. Care diversity and universality. A theory of nursing. Nursing and Health Care, 6/4: 208-212.

LOPEZ ALONSO, C. et al (1985) De la beneficencia al bienestar social: cuatro siglos de acción social. Consejo General de Colegios Oficiales de Diplomados en Trabajo Social y Asistentes Sociales. Siglo XXI, Madrid.

MALINOWSKI, B. (1984) Una teoría científica de la cultura. Sarpe, Madrid.

MESA, R. (1967) El colonialismo en la crisis del XIX español. Ciencia Nueva, Madrid.

ORGANIZACIÓN PARA LA COOPERACIÓN Y EL DESARROLLO ECONÓMICO (OCDE, 1998); Tendances des migrations internationales. Rapport annuel, París: OCDE.

PAJARES, M. (1998) La inmigración en España, Icaria, Madrid.

POSTMAN, N. (1983) La enseñanza como activi- 
dad crítica. Fontanella, Barcelona.

RAMÍREZ, Á. (1998) Migraciones, género e Islam. Agencia de Cooperación Española, Madrid.

SEMINARIO DE INVESTIGACIÓN PARA LA PAZ (1996) Cultura de la tolerancia. Seminario de Investigación para la Paz, Zaragoza.

SERVICIOS SOCIALES DE CATALUÑA (1985) Política social y servicios sociales. Marsiega, Madrid.

SILES, J et al (1993) Las alteraciones en la vida cotidiana de enfermos terminales a través del análisis de textos. Enfermería Científica, 131: 4-10.

SILES, J. (1995 ) Las características de los paradigmas científicos y su adecuación a la investigación en enfermería. Enfermería Científica, 160/161: 10-15.

-(1996) Pasado, presente y futuro de la enfermería en España. Perspectiva histórica y epistemo- lógica. CECOVA, Alicante.

-(1997) Epistemología y enfermería: por una fundamentación científica y profesional de la disciplina. Enfermería Clínica, 4/7:188-194.

-(1999) Historia de la enfermería comunitaria en España. Index, VIII/24-25: 25-31

(VV.AA.) (1990) Política de bienestar social para Euskadi en la Europa del 93. Departamento de Trabajo y Seguridad Social del Gobierno Vasco.

(VV.AA.) (1988) Relación entre servicios sociales y sanitarios. Consejo General de Colegios Oficiales de Diplomados en Trabajo Social y Asistentes Sociales/ Siglo XXI, Madrid.

WARE, C. (1968) Estudio de la comunidad: cómo averiguar recursos; cómo organizar esfuerzos. Humanitas, Buenos Aires.

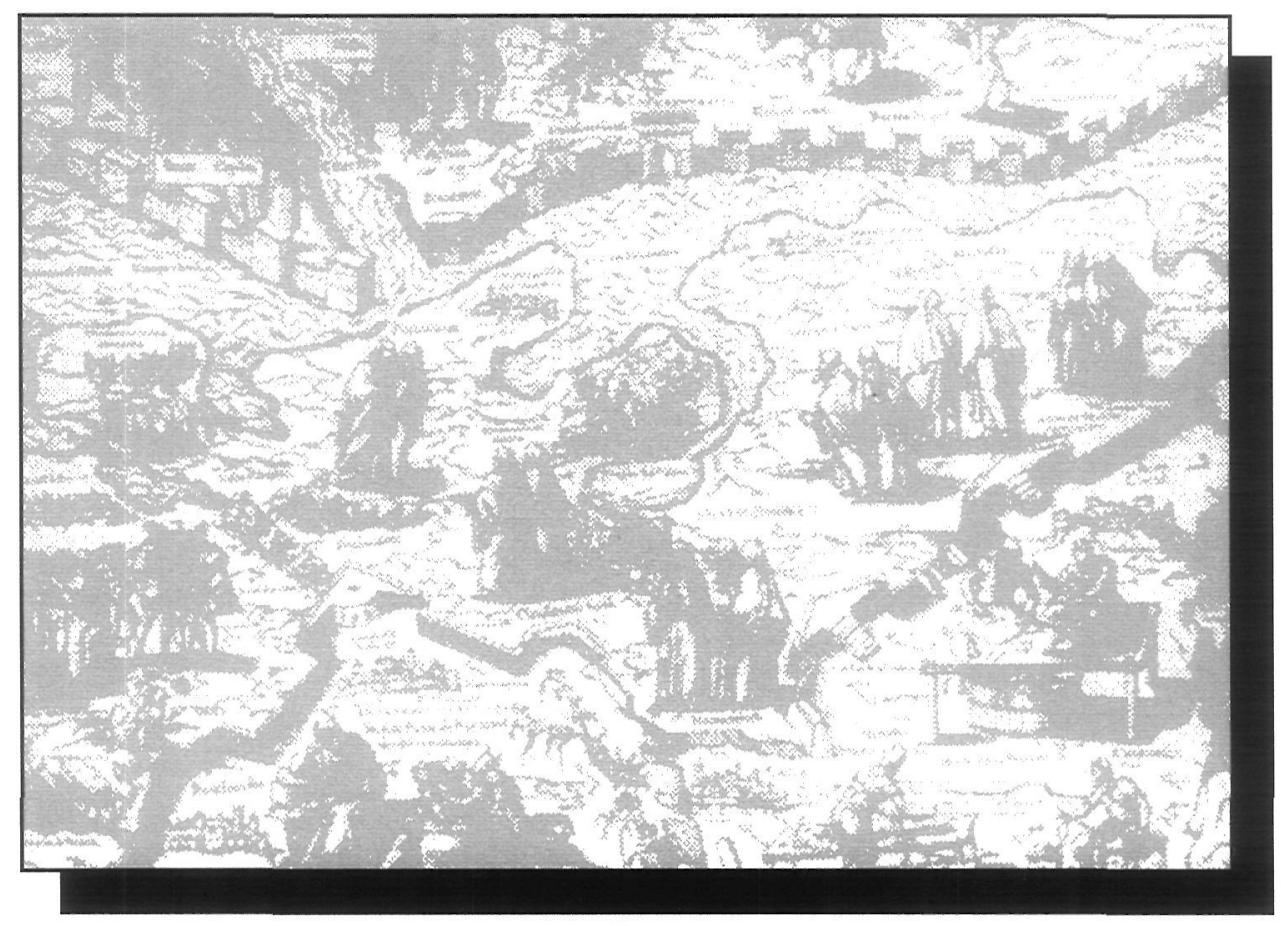

